\title{
Collective modes of an anisotropic quark-gluon plasma induced by relativistic jets
}

\author{
Mahatsab Mandal* \\ Saha Institute of Nuclear Physics, Kolkata, India \\ Government General Degree College At Kalna I, Burdwan, India \\ E-mail: mahatsab@gmail.com
}

\section{Pradip Roy}

Saha Institute of Nuclear Physics, Kolkata, India

E-mail: pradipk.royesaha.ac.in

\begin{abstract}
We present the study of the properties of the collective modes induced by the relativistic jets in an anisotropic quark-gluon plasma(AQGP). We assume that the partons in the jet can be assumed to have a colorless Tsunami-like momentum distribution. We derive and discuss the dispersion relation for both the stable and unstable modes of the composite system in the Vlasov approximation. We concentrate our calculation when the wave vector is parallel to the anisotropy direction as the filamentation instability of AQGP is maximum in this scenario. It is found that stable modes shift towards the light cone for nonzero values of the anisotropy parameter $(\xi)$ and the jet strength $(\eta)$. In the case of unstable mode, the growth rate depends on $\xi$ and $\eta$, and the nature of the growth rate depends on the jet velocity.
\end{abstract}

7th International Conference on Physics and Astrophysics of Quark Gluon Plasma

1-5 February, 2015

Kolkata, India

*Speaker. 


\section{Introduction}

In relativistic heavy-ion collision, jets with high transverse momentum travel through the hot and dense medium losing energy by collisional and radiative processes. This phenomenon is known as jet quenching, where high $p_{T}$ hadron production has been found to be strongly suppressed [1]. Apart from jet quenching, the jet particles also interact with the plasma leading to modified collective oscillation. This produces a color charge density wake and also wake potential $[2,3,4]$ in the QGP. The experimental evidence of the azimuthal dihadron correlation at RHIC shows a double peak structure in the away side [5] for the intermediate $p_{T}$ particles. Such peaks were predicted as a signature of Mach cones and Cerenkov-like radiation created by the partonic jets traveling through the QGP. Therefor, the interaction of a relativistic stream of charge particles with the plasma also influences the collective modes of the system. Within the framework of linear response theory, the interaction between the jet and the plasma shows an exponential growth of collective gauge fields, with a colorless tsunami-like initial momentum distribution of the jet $[6,7]$. The unstable modes arise at a velocity of the jet larger than the speed of sound in the QGP.

All of the phenomenological treatments are performed in situations where the distributions of the soft partons providing the thermal background are assumed to have isotropic momentum distributions. However, in the very early stage of heavy-ion collision, due to rapid longitudinal expansion at the onset of the QGP phase, anisotropy arises in the $p_{T}-p_{L}$ plane with $\left\langle p_{L}{ }^{2}\right\rangle<<$ $\left\langle p_{T}{ }^{2}\right\rangle$ in the local rest frame [8,9]. In studying the evolution of such a system, we use the method of the plasma physics within the framework of the quark-gluon transport theory[10] in weak coupling regime, i.e., $g<<1$. At this scale, we have neglected the hard mode interactions, assuming that the interactions between plasma and jet are only mediated by mean gauge fields.

We, in this work, will study a quantitative estimate of how the passage of the jet affects the dispersion relations [11] and the growth rate of the instabilities in AQGP.

\section{Self-Energy}

In this section, we shall briefly discuss how to calculate the polarization tensors in a purely anisotropic QGP system and in an AQGP with a jet propagating through it. We first discuss the structure of the polarization function in a purely AQGP i.e., in the absence of the jet. The spacelike component of the gluon polarization tensor of an anisotropic system can be written as

$$
\Pi_{p}^{i j}(K)=-g^{2} \int \frac{d^{3} p}{(2 \pi)^{3}} v^{i} \partial^{l} f(\mathbf{p})\left(\delta^{j l}+\frac{v^{j} k^{l}}{K \cdot V+i \varepsilon}\right)
$$

where $f(\mathbf{p})$ is an arbitrary distribution function. The anisotropic phase-space distribution function can be obtained from any isotropic distribution function by rescaling only in one direction in momentum space by changing the argument $f(\mathbf{p})=f_{\text {iso }}\left(\sqrt{\mathbf{p}^{2}+\xi(\mathbf{p} . \hat{\mathbf{n}})^{2}}\right)$. Here $f_{\text {iso }}$ is an arbitrary isotropic distribution function and $\hat{\mathbf{n}}$ is the direction of anisotropy. The parameter $\boldsymbol{\xi}$ is the degree of anisotropy $(-1<\xi<\infty)$. We can then decompose the self-energy into four structure functions as

$$
\Pi_{p}^{i j}(K)=\alpha A^{i j}+\beta B^{i j}+\gamma C^{i j}+\delta D^{i j}
$$


where

$$
A^{i j}=\delta^{i j}-k^{i} k^{j} / \mathbf{k}^{2}, \quad B^{i j}=k^{i} k^{j} / \mathbf{k}^{2}, \quad C^{i j}=\tilde{n}^{i} \tilde{n}^{j} / \tilde{n}^{2}, \quad D^{i j}=k^{i} \tilde{n}^{j}+k^{j} \tilde{n}^{i},
$$

with $\tilde{n}^{i}=A^{i j} n^{j}$, which obeys $\tilde{\mathbf{n}} . \mathbf{k}=0$ and $n^{2}=1$. The expressions for structure functions have been given in Ref. [11].

In order to study the collective modes due to the propagation of an energetic jet, we have to find also the structure of the self-energy induced by the jet. For simplicity, this can be done by assuming a colorless tsunami-like momentum distribution of the jet [7, 11]: $f_{\text {jet }}(\mathbf{p})=\bar{n} \bar{u}^{0} \delta^{(3)}(\mathbf{p}-\Lambda \overline{\mathbf{u}})$. Here $\bar{n}$ is a parameter proportional to the density; and $\bar{u}^{\mu}=\gamma\left(1, \mathbf{v}_{\mathrm{jet}}\right)$ is the four-velocity, where $\gamma$ is the Lorentz factor and $\mathbf{v}_{\text {jet }}$ is the velocity of the jet parton. Substituting the tsunamilike distribution function in Eq. 2.1 one deduces that

$$
\Pi_{\text {jet }}^{i j}(K)=\omega_{\text {jet }}^{2}\left(\delta^{i j}+\frac{k^{i} v_{\text {jet }}^{j}+k^{j} v_{\text {jet }}^{i}}{\omega-\mathbf{k} \cdot \mathbf{v}_{\mathrm{jet}}}-\frac{\left(\omega^{2}-\mathbf{k}^{2}\right) v_{\text {jet }}^{i} v_{\text {jet }}^{j}}{\left(\omega-\mathbf{k} \cdot \mathbf{v}_{\mathrm{jet}}\right)^{2}}\right)
$$

where $\omega_{\text {jet }}^{2}=\frac{g^{2} \bar{n}}{2 \Lambda}$ is the plasma frequency of the jet.

\section{Collective Modes}

We now consider the collective modes of the anisotropic plasma due to the propagation of an energetic jet. To do this, we note that in very short time regime, where the Vlasov approximation is valid, the polarization tensor of the composite system is additive, $\Pi_{t}^{\mu v}(K)=\Pi_{p}^{\mu v}(K)+\Pi_{\text {jet }}^{\mu v}(K)$. The dispersion relation for the collective modes of the total system can be found by solving the equation:

$$
\operatorname{det}\left[\left(k^{2}-\omega^{2}\right) \delta^{i j}-k^{i} k^{j}+\Pi_{t}^{i j}(K)\right]=0
$$

We define a dimensionless quantity $\eta=\omega_{\text {jet }}^{2} / \omega_{t}^{2}$, where $\omega_{t}^{2}=\omega_{\text {jet }}^{2}+m_{D}^{2} / 3$ which is related to Debye $\operatorname{mass}\left(m_{D}\right)$ and the plasma frequency of the jet. In the following subsection we analyze jet induced collective modes in AQGP for the two special cases, when the jet velocity is (a) perpendicular to the momentum i.e., $\mathbf{k} \perp \mathbf{v}_{\text {jet }}$ and (b) parallel to the momentum i.e., $\mathbf{k} \| \mathbf{v}_{\text {jet }}$.

\section{$3.1 \mathrm{k}$ orthogonal to $\mathrm{v}_{\text {jet }}$}

Here we choose the coordinates $\mathbf{k}=(0,0, k)$ and $\mathbf{v}_{\text {jet }}=\left(v_{\text {jet }}, 0,0\right)$. In this case the condition (3.1) reads as [11]:

$$
d_{22}\left(d_{11} d_{33}-d_{13}^{2}\right)=0
$$

The dispersion relation for all of the collective modes of the composite system can be determined by finding the solution to the equations, $d_{22}=0$ and $d_{11} d_{33}-d_{13}^{2}=0$ [11] which is referred to as mode I and mode II respectively. Collective mode I is independent of the jet velocity. However, it depends on the plasma frequency of the jet. The stable collective mode II shifts toward the light cone with the increase of $\xi$. Collective mode I and mode II also have poles along the imaginary $\omega$ axis. The dispersion relation for these modes can be calculated by taking $\omega=\omega_{0}+i \Gamma$ with $\omega_{0}$ and $\Gamma$ are real valued. Numerically we find that the collective modes are non propagating. At $\xi=0$ we do not find any unstable collective mode for mode I and the maximum value of the growth 

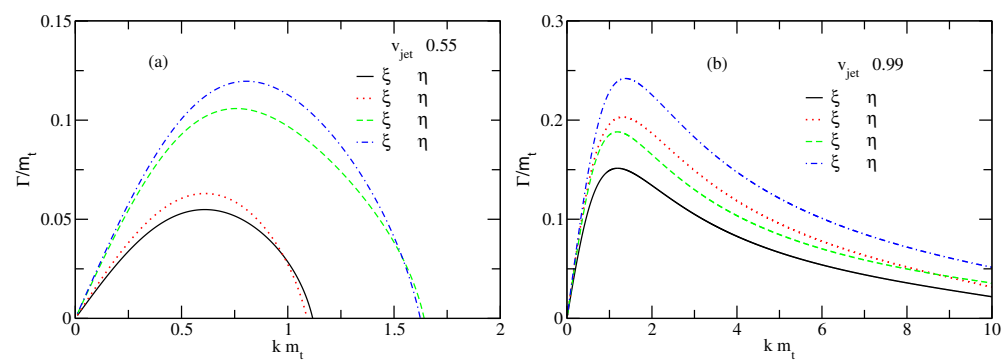

Figure 1: (Color online) Imaginary part of the dispersion relation of the unstable mode- II as a function of $k$ for two different values of the velocity of the jet with the variation of $\xi$ and $\eta$. The left(right) panel corresponds to $v=0.55(0.99)$.

rate increases with the strength of the anisotropy. In Figure (1) we present the imaginary part of the dispersion relation for the unstable mode II for two different velocities of the jet. With the increase of $\xi$ and $\eta, \Gamma_{\max }$ increases for both the jet velocities considered here. But the values of the momentum at which $\Gamma_{\max }$ occurs are independent of $\xi$ and $\eta$ at $v_{\text {jet }}=0.99$, as shown in Figure (1b). In order to find the wave number $k_{\max }\left(\xi, v_{\text {jet }}, \eta\right)$ at which the unstable modes of the spectrum terminates, we take the limit $\Gamma \rightarrow 0$ to obtain

$$
\left.k_{\text {max }}=\frac{\omega_{t}}{2 \sqrt{1-v_{\text {jet }}^{2}}}\left[3-2 v_{\text {jet }}^{2}(\eta-3)-7 \eta\right)-3(\eta-1)\left(-1+\xi+2 v_{\text {jet }}(1+\xi)\right) \frac{\arctan [\sqrt{\xi}]}{\sqrt{\xi}}\right]^{1 / 2} .
$$

\section{$3.2 \mathrm{k}$ parallel to $\mathrm{v}_{\text {jet }}$}
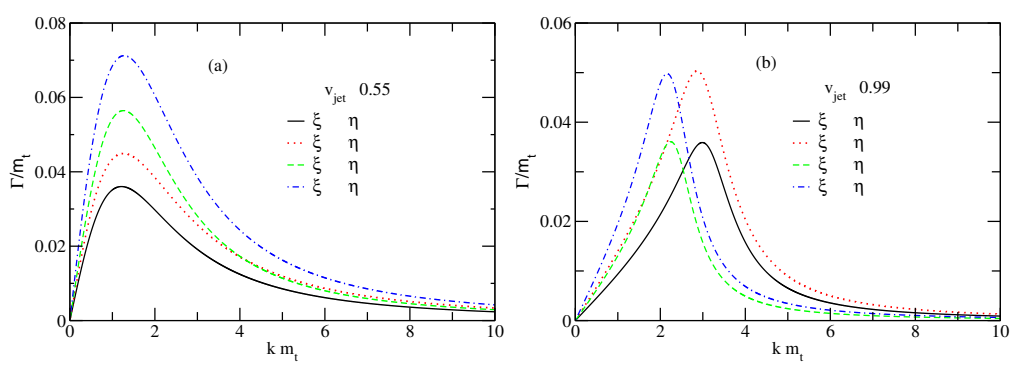

Figure 2: (Color online) Same as Fig. 1 for mode III

Next, we consider jet velocity is parallel to the momentum of the collective modes. and the corresponding solution of Eq.(3.1) is [11]

$$
d_{11}^{\prime} d_{22}^{\prime} d_{33}^{\prime}=0
$$

The solution of the dispersion relation shows that the collective modes of the composite system have three solutions. But we found $d_{11}^{\prime}$ and $d_{22}^{\prime}$ are same and identical modes as mode I. The dispersion relation for the other collective mode can be determined by solving $d_{33}^{\prime}=0$. Numerically we find that mode III is an unstable propagating mode. At $v_{\text {jet }}=0.55, \Gamma_{\max }$ increases with the 
increase of the anisotropy parameter $\xi$ and the strength of the jet $(\eta)$ as shown in Figure (2a). The value of the momentum corresponding to the maximum of $\Gamma$ is the same for all the cases considered here. Figure ( $2 b$ ) shows that the behavior of the growth rate is completely different when the value of the jet velocity is greater than the plasmon phase velocity. At fixed value of $\eta$, the maximum value of the growth rate $\Gamma$ does not change with $\xi$, but the value of the momentum is shifted toward the origin with the increase of $\xi$. At fixed value of $\xi$, the maximum value of $\Gamma$ increases with $\eta$ and the corresponding value of the momentum is approximately independent of $\eta$.

\section{Summary}

In this work, we have studied the effects of the jet of particles on the collective modes of an anisotropic QCD plasma using transport theory.To simplify the analysis, we assumed the initial distribution of the jet of particles a colorless tsunami-like distribution. We have presented the collective modes for two spacial cases. For the case when $\mathbf{k} \perp \mathbf{v}_{\text {jet }}$, we find two stable modes (I and II), of which one is independent of the jet velocity. In case of $\mathbf{k} \| \mathbf{v}_{\text {jet }}$ we obtain two identical modes as mode I and one distinct mode III. For case of mode II, $\Gamma_{\max }$ increases with the velocity of the jet. With the increase of $\eta$ or $\xi, \Gamma_{\max }$ also increases for both the jet velocities considered here. For mode III, $\Gamma_{\max }$ is independent of $\xi$, when the jet velocity is greater than the phase velocity of plasmon at fixed $\eta$. Numerically, we find the maximum value of the growth rate of such system runs between $\Gamma_{\max } \sim(0.05-0.25) \omega_{t}$.

\section{References}

[1] M. Gyulassy, P. Levai and I. Vitev, Jet quenching in thin quark-gluon plasmas I: formalism, Nucl. Phys. B 571, 197(2000).

[2] J. Ruppert and B. Muller, Waking the colored plasma, Phys. Lett. B 618, 123 (2005).

[3] M. Mandal and P. Roy, Wake in anisotropic quark-gluon plasma, Phys. Rev. D 86, 114002 (2012).

[4] M. Mandal and P. Roy, Wake potential in collisional anisotropic quark-gluon plasma, Phys. Rev. D 88, 074013 (2013).

[5] S. S. Adler et al. (PHENIX Collaboration), Dense-Medium Modifications to Jet-Induced Hadron Pair Distributions in $A u+A u$ Collisions at $\sqrt{S_{N N}}=200 \mathrm{GeV}$, Phys. Rev. Lett. 97, 052301 (2006).

[6] M. Mannarelli and C. Manuel, Chromohydrodynamical instabilities induced by relativistic jets, Phys. Rev. D 76, 094007 (2007).

[7] M. Mannarelli and C. Manuel, Jet-induced gauge field instabilities in the quark-gluon plasma: A kinetic theory approach, Phys. Rev. D 77, 054018 (2008).

[8] M. Martiez and M. Strickland, Measuring Quark-Gluon-Plasma Thermalization Time with Dileptons, Phys. Rev. Lett. 100, 102301 (2008).

[9] M. Mandal, P. Roy, Some Aspects of Anisotropic Quark-Gluon Plasma, Adv.High Energy Phys. 2013, 1 (2013).

[10] S. Mrowczynski, Color collective effects at the early stage of ultrarelativistic heavy-ion collisions, Phys. Rev. C 49, 2191 (1994).

[11] M. Mandal and P. Roy, Jet-induced collective modes in an anisotropic quark-gluon plasma, Phys. Rev. D 89, 074016 (2014). 